\title{
Dynamic lot sizing problem under uncertainty of returned products in remanufacturing industry
}

\author{
Iman Setyoaji ${ }^{1, *}$ \\ ${ }^{1}$ Department of Industrial Engineering, Faculty of Engineering Universitas Indonesia, 16424 Depok, \\ Indonesia
}

\begin{abstract}
Remanufacturing processes face uncertainty in the quality of the items being returned by customers, this significant variability complicates the control of inventories. Demands can be satisfied by procured new items, but also by remanufactured returned items. This paper develops dynamic lot sizing model for remanufacturing industry under uncertainty of returned items and proposes Bayesian Inference to estimate the replacement ratio of returned items that used to determine those lot sizes for new items. The objective of this paper is to minimize the total cost composed of holding cost and set-ups cost. A numerical example is provided based on case study. The result shows that total cost is reduced to be $45 \%$.
\end{abstract}

\section{Introduction}

In recent years, issues on energy conservation and environmental protection have been the main focus for many countries and international organizations due to the depletion of resource reserves and increased environmental pollution. Every manufacturing industry is required to commit to reduce the environmental impact of production processes through the efficient use of resources [1]. The remanufacturing system is an industry that can be contributed in reducing waste hazards and also more economically due to the production cost are cheaper than the manufacturing systems [2].

Remanufacturing is the process of returning the condition of used products into high quality. There are several stages in remanufacturing systems, including: collecting of used products, disassembling into several items, cleaning and checking each items, and the last is reassembling of the items and tested so it is as good as new.

Lot-sizing problems have attracted the attention of

researchers. The dynamic lot size is one of the most studied topics. The dynamic lot size model is a generalization of the Economic Order Quantity (EOQ) model that takes into account that demand for the product varies over time. This model was introduced by Wagner and Whitin (1958). There are lots of the scientific literature addresses the dynamic

*Corresponding author: iman.setyoaji@gmail.com 
lot size in manufacturing systems. However discussing about dynamic lot size problem in remanufacturing system still rarely appear. Teunter et al. (2006) introduce a dynamic lot sizing model with returns and remanufacturing and distinguish between the case of joint and seperate setup cost for the replenishment sources remanufacturing and manufacturing.

They test in a large numerical experiment three well-known heuristic approaches that were adapted from the single-item dynamic lot sizing problem without returns. In both model settings, the Silver-Meal based heuristic has been shown to be the best heuristic resulting in an average deviation of 3\% from the optimal solution in the joint and $8.3 \%$ in the seperate setup cost setting. Using heuristics to handle these problems has been motivated by the fact that the authors conjecture the underlying problem of the mseperate setup cost setting to be NP-hard [3].

Pan et al. (2009) extend the analysis of Teunter et al. (2006) by including a disposal option for returned products and by restricting production, remanufacturing, and disposal capacities. They illustrate different problem formulations and elaborate dynamic programming algorithms to solve some of these problems to optimality [4].

Schulz (2009) modifies the SM heuristic discussed in Teunter et al. (2006) by applying methods elaborated for the corresponding static problem and attaching two simple improvement steps that reduce the error gap noticeably [5]. He studies in original equipment manufactur (OEM) that sells one product to his customers over a planning horizon of $\mathrm{T}$ periods.

This work proposes a generalization of dynamic lot sizing model introduced by Schulz (2009) and implements it in independently operating remanufacturer (remanufacturing industry) by considering a two-period model in determining lot sizes for new items and the replacement ratio of returned items is estimated by Bayesian Inference to determine lot sizes for new items in the first period. The remainder of this contribution is organized as follows. Section 2 introduces the basic modelling assumptions. Section 3 introduces the generalization of dynamic lot sizing model introduced by Schulz (2009) and the calculation of Bayesian Inference are depicted in this chapter. The result of this study can be found in Section 4. Finally, Section 5 presents a short summary and an outlook on future research opportunities.

\section{Basic Modelling Assumptions}

In his contribution, Schulz (2009) introduced a dynamic lot sizing model by modifying the SM heuristic discussed in Teunter et al (2006) by proposing additional improvements that reduce the error gap noticeably. The basic assumptions of this modelling approach can be outlined as follows. As depicted in Figure 1, the independently operating remanufacturer (IO) is considered that sell remanufacturing product to his customers over a planning horizon of $T$ periods.

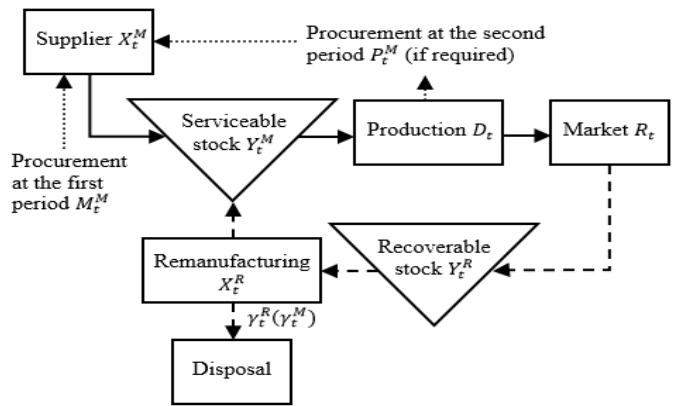

Fig. 1. Dynamic lot sizing model in independently operating remanufacturer/remanufacturing industry 
In each period $t=1, \ldots, T$ his customers demand a discrete and known amount of this product which will be further on denoted by $D_{t}$. The independently operating remanufacturer/remanufacturing industry provides each customer the opportunity to return her/his product if it is broken or when she/he has no further use for it. All returns that pass the inspection (which will denoted by $R_{t}$ ) are brought to a recoverables stock $Y_{t}^{R}$. Per unit time a recoverable item incurs holding costs of $h^{R}$.

Prior to Remanufacture the products which will know the lot sizes of remanufacturing items $X_{t}^{R}$ in period $t$ (by paying the set costs $K^{R}$ ), the new items should be procured in the first period $X_{t}^{M}$ to replenish the defective items that will be known after disassembling process (setting up a new items lot in period $t$ incurs fixed costs $K^{M}$ while holding a serviceable item for one period in the respective inventory costs $h^{M}$ ). Therefore, lot sizes for new items is calculed based on the replacemet ratio of defective parts. Both of remanufacturing items and new items are brought to a serviceable stocks $Y_{t}^{R}$. If sum of remanufacturing items and new items are not equal to demand, procurement of new items in the second period $P_{t}^{M}$ should be provided. Next to the notation introduced above two more decision variables are required. If a remanufacturing lot is initiated in period $t$ (i.e. $X_{t}^{R}=0$ ) the binary decision variable $\gamma_{t}^{R}$ becomes one. However, if $X_{t}^{R}=0$ the decision variable $\gamma_{t}^{R}$ becomes zero. Likewise, $\gamma_{t}^{M}$ is set to one when a new item is procured in period $t$ and to zero if no item need to be procured. The model can be formulated as:

$$
\begin{aligned}
& \text { Min } \sum_{t=1}^{T}\left\{K^{R} \gamma_{t}^{R}+K^{M} \gamma_{t}^{M}+h^{R} y_{t}^{R}+h^{M} y_{t}^{M}\right\} \\
& y_{t}^{R}=y_{t-1}^{R}+R_{t}-X_{t}^{R} \quad \forall t=1,2, \ldots, N \\
& y_{t}^{M}=y_{t-1}^{M}+X_{t}^{R}+X_{t}^{M}-D_{t} \quad \forall t=1,2, \ldots, N \\
& X_{t}^{M}=P_{t}^{M}+M_{t}^{M} \quad \forall t=1,2, \ldots, N \\
& M_{t}^{M}=\varepsilon \cdot D t \quad \forall t=1,2, \ldots, N \\
& P_{t}^{M}=\left(D t-M_{t}^{M}-X_{t}^{M}\right) \gamma_{t}^{M} \quad \forall t=1,2, \ldots, N \\
& X_{t}^{R} \leq Q \cdot \gamma_{t}^{R} \quad \forall t=1,2, \ldots, N \\
& X_{t}^{M} \leq Q \cdot \gamma_{t}^{M} \quad \forall t=1,2, \ldots, N \\
& X_{0}^{R}=X_{0}^{M}=0 \quad \forall t=1,2, \ldots, N
\end{aligned}
$$

The objective function (1) minimizes the sum of all relevant setup and holding costs. Constraints (2) and (3) represent inventory balance equations that describe the inventory at the end of period $t$ as the inventory at the beginning of this period plus its inflows and minus its outflows. Restriction (4) represents lot sizes for new items which depended on the numbers of item procured at the first and second period. Constrains (5) procurement at the firts period should be provid, lot sizes of this new item is based on the replacement ratio of defective part $\varepsilon$. Constrains (6) procurement at the second period when the demand is not satisfied (if required). In order to ensure that fixed costs have to be paid whenever a lot is scheduled restrictions (7) and (8) have to be established whereas Q needs to be a 
sufficiently large number (e.g. the sum of all demands during the planning horizon). Finally, non-negativity and binary constraints have to be defined as well on the decision variable.

\section{Numerical Experiments}

In this section, replacement ratio of defective part is estimated by Bayesian Inference. The result of the estimation will be used to determinde lot sizes of new items at the first period.

\subsection{Computation of Replacement Ratio}

The author is interested in learning about the defective of cylinder head in $12 \mathrm{~V}$ diesel engine that returned from customer. The number's of cylinder head in one engine is 12 items. The production manager informs that the replacement ratio of cylinder head returned in one job order (one engine) is $77 \%$. Here we think of a population of cylinder head production and let $p$ represent the proportion of this population which damage.

The value of the proportion $p$ is unknown. In the Bayesian viewpoint, a person's beliefs about the uncertainty in this proportion are represented by a probability distribution placed on this parameter. This distribution reflects the person's subjective prior opinion about plausible values of $p$.

A random sample of cylinder heads from a particular job order will be taken to learn about this proportion. But first the researcher does some initial research to learn about the cylinder head as shown Table 1 below:

Table 1. Results of Disassembling Cylinder head

\begin{tabular}{ccccccccccc}
\hline Period & $\begin{array}{c}\text { No's of } \\
\text { job order }\end{array}$ & $\begin{array}{c}\text { No's of } \\
\text { cylinder } \\
\text { head }\end{array}$ & $\begin{array}{c}\text { No's of } \\
\text { damage }\end{array}$ & Crack & Rush & Chipping Scratch & Worn & Broken & Limit \\
\hline \hline Jan'18 & 26 & 312 & 162 & $19 \%$ & $76 \%$ & $2 \%$ & $0 \%$ & $0 \%$ & $2 \%$ & $1 \%$ \\
Feb'18 & 17 & 204 & 120 & $37 \%$ & $44 \%$ & $5 \%$ & $2 \%$ & $8 \%$ & $0 \%$ & $4 \%$ \\
\hline
\end{tabular}

Based on this information, the researcher believes that the cylinder heads returned from customer generally get damage and so $p$ (the proportion that cylinder head damage is .6 that is, it is equally likely that $p$ is smaller than .6 , or $p$ is larger than .6. and he is pretty confident (with probability .9) that cylinder head damage proportion is smaller than .75. In other words, the prior median is .75 and the prior 9 th precentile is .75 .

A sample of 14 job order is taken (it means no's of cylinder head is 168), 60 record that the cylinder head is damage. Based on the prior information and these observed data, the researcher is interested in estimating the proportion $p$.

Suppose that the prior density for $p$ is denoted by $g(p)$. If we regard a "success" as damage and we take a random sample with $s$ successes and $f$ failures, than the likelihood function is given by

$$
L(p) \propto p^{s}(1-p)^{f}, 0<p<1
$$

The posterior density for $p$, by Bayes' rule, is obtained, up to proportionality constant, by multiplying the prior density by likelihood:

$$
g(p \mid \text { data }) \propto g(p) L(p)
$$

Since the proportion is a continuous parameter, an alternative approach is to construct a density $g(p)$ on the interval $(0,1)$ that represents the person's initial beliefs. The function 
beta.select in the LearnBayes package is useful for finding the shape parameters of the beta density that match this prior knowledge. The inputs to beta.select are two lists, quantile1and quantile2, that define these two prior percentiles, and the function returns the values of the matching beta parameters.

$>$ library(LearnBayes)

$>$ quantile $2=\operatorname{list}(\mathrm{p}=.9, \mathrm{x}=.75)$

$>$ quantile $1=\operatorname{list}(\mathrm{p}=.5, \mathrm{x}=.6)$

$>$ beta.select(quantile1,quantile2)

[1] 9.326 .32

The prior information is matched with the beta density with $a=9.32$ and $b=6.32$. Combining this beta prior with the likelihood function, one can show the posterior density is also of the beta form with updated parameters $a+s$ and $b+f$ ).

$$
g(p \mid \text { data }) \propto p^{a+s-1}(1-p)^{b+f-1}, 0<p<1
$$

Where $a+s=9.32+60$ and $b+f=6.32+108$. Since the prior, likelihood, and posterior are all in the beta family, $\mathrm{R}$ command dbeta is used to compute the values of the prior, likelihood, and posterior.

$>\mathrm{a}=9.32$

$>\mathrm{b}=6.32$

$>\mathrm{s}=60$

$>\mathrm{f}=108$

$>\operatorname{curve}(\operatorname{dbeta}(\mathrm{x}, \mathrm{a}+\mathrm{s}, \mathrm{b}+\mathrm{f})$, from $=0$, to $=1$,

+ xlab="p",ylab="Density",lty=1,lwd=4)

$>$ curve $(\operatorname{dbeta}(\mathrm{x}, \mathrm{s}+1, \mathrm{f}+1)$, add $=$ TRUE, $1 \mathrm{ty}=2, \mathrm{lwd}=4)$

$>$ curve $(\operatorname{dbeta}(\mathrm{x}, \mathrm{a}, \mathrm{b}), \mathrm{add}=\mathrm{TRUE}, \mathrm{lty}=3, \mathrm{lwd}=4)$

$>$ legend(.7,7,c("Prior","Likelihood","Posterior"),

+ lty $=\mathrm{c}(3,2,1), \operatorname{lwd}=\mathrm{c}(3,3,3))$

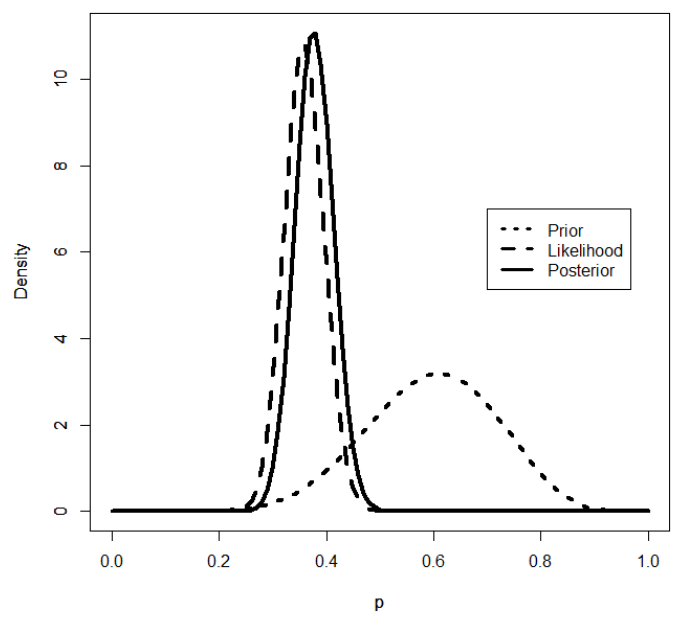

Fig. 2. Prior Density $g(p)$, Fungsi Likelihood $L(p)$, And The Posterior Density $g(p \mid$ data) 
A $90 \%$ interval estimate for $p$ is found by computing the 5th and 95 th percentiles of the beta density:

$>\operatorname{qbeta}(\mathrm{c}(0.05,0.95), \mathrm{a}+\mathrm{s}, \mathrm{b}+\mathrm{f})$

[1] 0.31954590 .4369310

A $90 \%$ posterior credible interval for the proportion is $(0.319,0.436)$.

An alternative method of summarization of a posterior density is based on simulation. In this case, we can simulate a large number of values from the beta posterior density and summarize the simulated output. Using the random beta command rbeta, we simulate 1000 random proportion values from the beta $(a+s, b+f)$ posterior by using the command,

ps $=\operatorname{rbeta}(1000, a+s, b+f)$

and display the posterior as a histogram of the simulated values in Figure

hist(ps,xlab="p",main="")

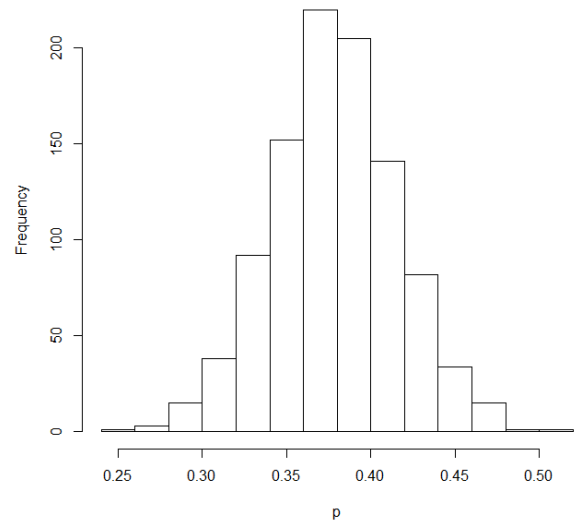

Fig. 3. Simulated samples of posterior beta distribution $p$

A $90 \%$ interval estimate can be estimated by the 5th and 95 th sample quantiles of the simulated sample.

quantile(ps, c $(0.05,0.95))$

$5 \% \quad 95 \%$

0.31825190 .4401859

\subsection{Computation of Lot Sizes}

In calculating the lot size, sample data is taken by observing the disassembly process in production area and Part Recommended System (PRS) data in material control area. The amount of observed data refers to the production planning in which the number of engines disassembled is $14 \mathrm{Job}$ Order $(\mathrm{JO})$ on the $12 \mathrm{~V}$ type engine. Since the number of cylinder heads on the $12 \mathrm{~V}$ type engine is 12 items, the total cylinder head is $14 \times 12=168$ (it means, the number of cylinder heads returned is equal to 168 . In the observation of 168 cylinder head, 60 cylinder head are damaged or can not be reused This judgment refers to the 
process of inspection and quality assessment applicable to the company The amount of damage to the cylinder head can be seen in detail in the following table:

Table 2. Cylinder Head Damage

\begin{tabular}{|c|c|c|c|c|c|c|c|c|c|c|c|c|c|c|}
\hline \multirow{2}{*}{ No. } & \multirow{2}{*}{ Job Order } & \multicolumn{10}{|c|}{ Cylinder Head ke- } & \multirow{2}{*}{ Total } \\
\cline { 2 - 13 } & $\mathbf{1}$ & $\mathbf{2}$ & $\mathbf{3}$ & $\mathbf{4}$ & $\mathbf{5}$ & $\mathbf{6}$ & $\mathbf{7}$ & $\mathbf{8}$ & $\mathbf{9}$ & $\mathbf{1 0}$ & $\mathbf{1 1}$ & $\mathbf{1 2}$ & \\
\hline & $\mathbf{1 1 1 8 0 1 1 7}$ & 1 & 1 & 1 & 1 & 1 & 1 & 1 & 1 & 1 & 1 & 0 & 0 & 10 \\
\hline & $\mathbf{1 1 1 8 0 1 0 9}$ & 1 & 0 & 1 & 1 & 0 & 1 & 0 & 1 & 1 & 0 & 0 & 0 & 6 \\
\hline & $\mathbf{1 1 1 8 0 1 1 2}$ & 0 & 0 & 0 & 0 & 1 & 1 & 0 & 0 & 0 & 0 & 0 & 0 & 2 \\
\hline & $\mathbf{1 1 1 7 0 6 9 4}$ & 1 & 1 & 1 & 1 & 1 & 0 & 0 & 0 & 0 & 1 & 1 & 1 & 8 \\
\hline & $\mathbf{6 1 1 7 0 0 0 1}$ & 0 & 1 & 0 & 1 & 1 & 1 & 0 & 1 & 1 & 1 & 0 & 0 & 7 \\
\hline & $\mathbf{6 1 1 7 0 0 0 2}$ & 0 & 0 & 0 & 0 & 0 & 0 & 0 & 0 & 0 & 1 & 0 & 1 & 2 \\
\hline & $\mathbf{6 1 1 7 0 0 0 5}$ & 0 & 0 & 0 & 0 & 0 & 0 & 0 & 0 & 0 & 0 & 0 & 0 & 0 \\
\hline & $\mathbf{1 1 1 7 0 5 7 4}$ & 0 & 0 & 0 & 1 & 0 & 0 & 0 & 1 & 0 & 0 & 0 & 0 & 2 \\
\hline & $\mathbf{1 1 1 7 0 6 1 4}$ & 1 & 1 & 0 & 0 & 1 & 1 & 0 & 1 & 1 & 1 & 0 & 0 & 7 \\
\hline & $\mathbf{1 1 1 8 0 1 1 1}$ & 1 & 1 & 0 & 0 & 0 & 0 & 0 & 0 & 0 & 0 & 1 & 1 & 4 \\
\hline & $\mathbf{1 1 1 7 0 6 6 2}$ & 1 & 1 & 0 & 1 & 0 & 0 & 1 & 0 & 1 & 1 & 0 & 1 & 7 \\
\hline & $\mathbf{1 1 1 8 0 0 7 0}$ & 0 & 0 & 0 & 0 & 0 & 0 & 0 & 0 & 0 & 0 & 0 & 0 & 0 \\
\hline & $\mathbf{1 1 1 7 0 7 6 7}$ & 0 & 0 & 0 & 0 & 0 & 0 & 0 & 1 & 1 & 1 & 1 & 1 & 5 \\
\hline & $\mathbf{1 1 1 7 0 7 1 8}$ & 0 & 0 & 0 & 0 & 0 & 0 & 0 & 0 & 0 & 0 & 0 & 0 & 0 \\
\hline & & & & & & & & & & & & & & $\mathbf{6 0}$ \\
\hline
\end{tabular}

In the calculation, the lot size is equal to the number of new parts that have been ordered through the MRP or PRS system. Calculations were performed with two different change ratios of $44 \%$ and $77 \%$. The ratio of $44 \%$ is obtained from the calculation using Bayesian approach, while $77 \%$ is the current replacement ratio in the company. This replacement ratio is used to determine the number of parts ordered through the MRP. It is assumed that inventory cost is as shown in table below, than the total inventory cost can be calculated.

Table 3. Inventory costs

\begin{tabular}{|l|c|}
\hline Remanufacturing & 0,6 \\
\hline Holding cost $(\mathrm{Hr})$ & 20 \\
\hline Setup cost $(\mathrm{Kr})$ & \\
\hline New Parts & 1 \\
\hline Holding cost $(\mathrm{Hs})$ & 40 \\
\hline Setup cost $(\mathrm{Ks})$ & \\
\hline
\end{tabular}

In this paper, the researcher compute the lot size on Ms.Excel 2007. The formulation of calculation is shown the below figure:

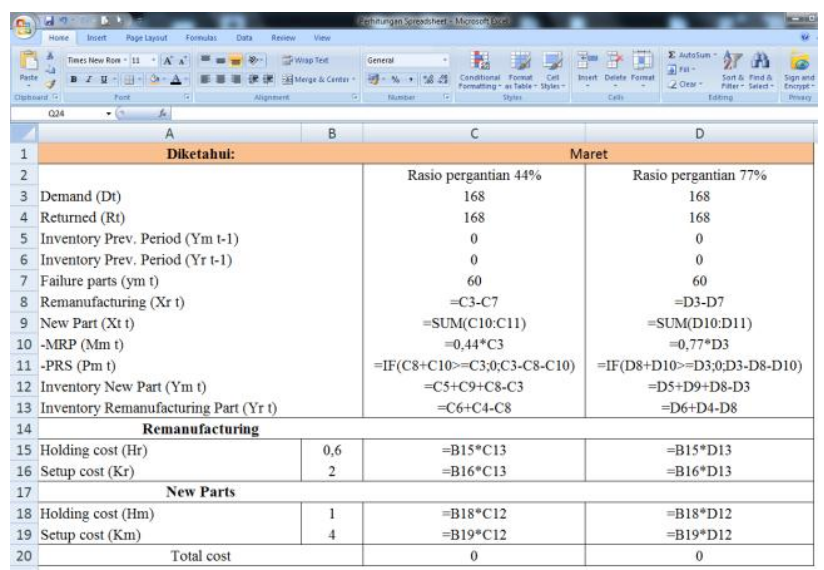

Fig. 4. Formulation of invetory cost and lot sizing calculation 
The calculation result is shown the below figure :

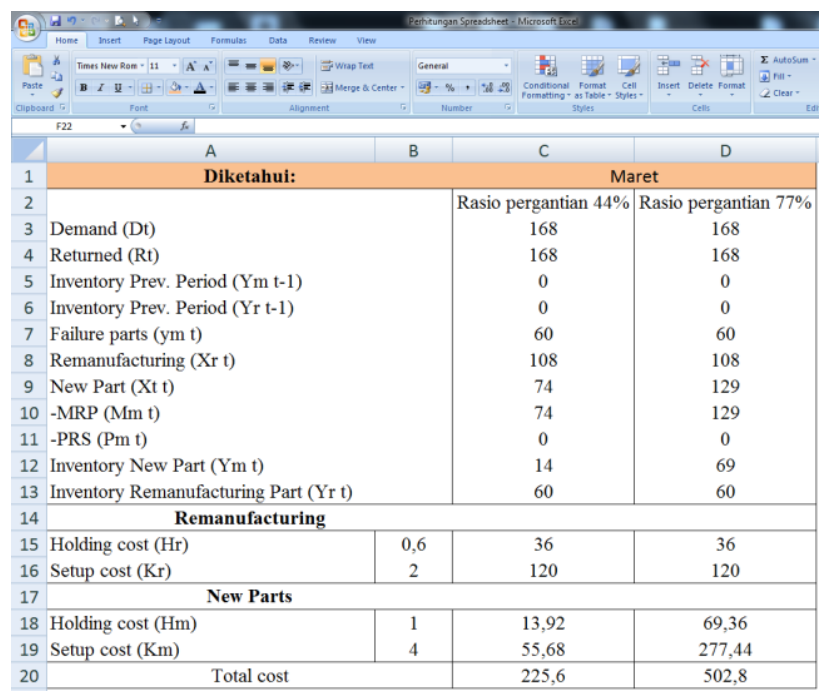

Fig. 5. The result of inventory cost and lot sizing calculatio

The number of product returned is 168 items and the remanufacturing items are 108 items or the number of broken components is 60 items, Due to Remanufacturing processes face uncertainty in the quality of the items, we can can not make sure how many cylinder head damage, it is posibility less than or more than 60 items. Extreme possibilities can happen, for example damage equal to 0 items or 168 items. The replacement ratio determines the size of the lot ordered through the MRP system. Mistakenly in calculation of it will impact the lot size to be ordered. It will increase or decrease storage costs (holding costs) as well as set-up costs. From the calculation result, the total inventory cost with the turnover ratio is $44 \%$ lower than the total inventory cost with a turnover ratio of $77 \%$. This is because, the quantity of new components with a $44 \%$ turnover ratio stored in the warehouse is lower.

\section{Conclusion}

Determination of dynamic lot size has been obtained through model developing and applied data according to case study. The conceptual model developed successfully minimizes total inventory costs to be $45 \%$.

The conceptual model developed is used in the remanufacturing industry. The degree of uncertainty in the remanufacturing industry is very high because the quality and quantity of product returns are identified.

The replacement ratio determines the quantity of the newly ordered component lot. Bayesian inference is a good method for extracting information from data with uncertainty. It is very useful to calculate the replacement ratio.

This study has a limitation of subjectivity at the time of the study. Researchers use their beliefs to make the best guess at the time of the prior determination. Future research efforts can be directed in the prior determination. 


\section{References}

1. J. W. Sutherland, D. P. Adler, K. R. Haapala, \& V. Kumar, Manufacturing Technology, 57, 5-8. (2008)

2. H. Yanting, \& W. Zongjung, J. Cle Prod. 142, 3917-3927 (2017)

3. H. Yanting \& W. Zongjung, J. Cle Prod. 142, 3917-3927 (2017)

4. R. H. Teunter, Z. P. Bayindir and W. van den Heuvel, IJPR, 44 (20), 4377-4400 (2006)

5. Z. Pan, J. Tang and O, Liu, EJOR, 198 (3), 810-821. (2009)

6. T. A. Schulz, IJPR, 49(9):2519-2533. (2009) 